\title{
THE IDEAL THEORY IN QUOTIENTS OF COMMUTATIVE SEMIRINGS
}

\author{
Shahabaddin EBrahimi Atani \\ University of Guilan, Iran
}

\begin{abstract}
Since the theory of ideals plays an important role in the theory of quotient semirings, in this paper, we will make an intensive study of the notions of Noetherian, Artinian, prime, primary, weakly primary and $k$-maximal ideals in commutative quotient semirings. The bulk of this paper is devoted to stating and proving analogues to several well-known theorems in the theory of the residue class rings.
\end{abstract}

\section{INTRODUCTION}

The concept of semirings was introduced by H. S. Vandiver in 1935 and has since then been studied by many authors (see, for example, [2], [3], [5], [6], [7]). This paper is concerned with generalization of some results of factor ring theory to quotient semiring theory. A number of results concerning prime, weakly prime, primary, weakly primary, maximal and $k$-maximal ideals of such semirings are given (see section 2 ).

For the sake of completeness, we state some definitions and notations used throughout. A commutative semiring $R$ is defined as an algebraic system $(R,+, \cdot)$ such that $(R,+)$ and $(R, \cdot)$ are commutative semigroups, connected by $a(b+c)=a b+a c$ for all $a, b, c \in R$, and there exists $0 \in R$ such that $r+0=r$ and $r 0=0 r=0$ for each $r \in R$. In this paper all semirings considered will be assumed to be commutative semirings. A semiring $R$ is said to be a semidomain if $a b=0(a, b \in R)$, then either $a=0$ or $b=0$. A semifield is a semiring in which non-zero elements form a group under multiplication.

A subset $I$ of a semiring $R$ will be called an ideal if $a, b \in I$ and $r \in R$ implies $a+b \in I$ and $r a \in I$. A subtractive ideal (= $k$-ideal) $K$ is an ideal

2000 Mathematics Subject Classification. 16Y60.

$K e y$ words and phrases. Semiring, $k$-ideals, $Q$-ideals, quotient semiring. 
such that if $x, x+y \in K$ then $y \in K$ (so $\{0\}$ is a $k$-ideal of $R$ ). A prime ideal of $R$ is a proper ideal $P$ of $R$ in which $x \in P$ or $y \in P$ whenever $x y \in P$. So $P$ is prime if and only if $A$ and $B$ are ideals in $R$ such that $A B \subseteq P$, then $A \subseteq P$ or $B \subseteq P$ where $A B=\{a b: a \in A$ and $b \in B\}$ (see [3, Theorem 5]). A primary ideal of $P$ of $R$ is a proper ideal of $R$ such that, if $x y \in P$ and $x \notin P$, then $y^{n} \in P$ for some positive integer $n$.

An ideal $I$ of a semiring $R$ is called a partitioning ideal (= $Q$-ideal) if there exists a subset $Q$ of $R$ such that

(1) $R=\cup\{q+I: q \in Q\}$

(2) If $q_{1}, q_{2} \in Q$ then $\left(q_{1}+I\right) \cap\left(q_{2}+I\right) \neq \emptyset$ if and only if $q_{1}=q_{2}$.

Let $I$ be a $Q$-ideal of a semiring $R$ and let $R / I=\{q+I: q \in Q\}$. Then $R / I$ forms a semiring under the binary operations $\oplus$ and $\odot$ defined as follows:

$$
\left(q_{1}+I\right) \oplus\left(q_{2}+I\right)=q_{3}+I
$$

where $q_{3} \in Q$ is the unique element such that $q_{1}+q_{2}+I \subseteq q_{3}+I$.

$$
\left(q_{1}+I\right) \odot\left(q_{2}+I\right)=q_{4}+I
$$

where $q_{4} \in Q$ is the unique element such that $q_{1} q_{2}+I \subseteq q_{4}+I$. This semiring $R / I$ is called the quotient semiring of $R$ by $I$. By definition of $Q$-ideal, there exists a unique $q^{\prime} \in Q$ such that $0+I \subseteq q^{\prime}+I$. Then $q^{\prime}+I$ is a zero element of $R / I$. Clearly, if $R$ is commutative, then so is $R / I$ (see $[5,6]$ ).

\section{QuOTIENT OF SEMIRINGS}

Our starting point is the following lemma:

Lemma 2.1. Let $R$ be a semiring, $I$ a $Q$-ideal of $R$ and $A$ a $k$-ideal of $R$ with $I \subseteq A$. Then $I$ is a $A \cap Q$-ideal of $A$.

Proof. It suffices to show that $A=\cup\{q+I: q \in Q \cap A\}$. Since $\cup\{q+I: q \in Q \cap A\} \subseteq A$ is trivial, we will prove the reverse inclusion. Suppose that $x \in A$. Then $x \in q+I$ for some $q \in Q$; hence there is an element $a$ of $I \subseteq A$ such that $x=q+a$. Then $q \in A \cap Q$ since $A$ is a $k$-ideal, so we have equality.

Proposition 2.2. Let $R$ be a semiring, $I$ a $Q$-ideal of $R$ and $A$ a $k$-ideal of $R$ with $I \subseteq A$. Then $A / I=\{q+I: q \in A \cap Q\}$ is a $k$-ideal of $R / I$.

Proof. Let $q^{\prime}$ be the unique element in $Q$ such that $q^{\prime}+I$ is the zero in $R / I$. First, we show that $q^{\prime}+I \in A / I$. Let $a+I \in A / I \subseteq R / I$ where $a \in A \cap Q$. Then $(a+I) \oplus\left(q^{\prime}+I\right)=a+I$ where $a+q^{\prime}+I \subseteq a+I$, so $a+q^{\prime}+e^{\prime}=a+f^{\prime}$ for some $e^{\prime}, f^{\prime} \in I$; hence $q^{\prime} \in A \cap Q$ since $A$ is a $k$-ideal of $R$. Thus $q^{\prime}+I \in A / I$. Next, suppose that $\overline{q_{1}}=q_{1}+I, \overline{q_{2}}=q_{2}+I \in A / I$ where $q_{1}, q_{2} \in A \cap Q$. There is a unique element $q_{3} \in Q$ with $\overline{q_{1}} \oplus \overline{q_{2}}=q_{3}+I$ and $q_{1}+q_{2}+I \subseteq q_{3}+I$, so $q_{1}+q_{2}+e=q_{3}+f \in A$ for some $e, f \in I$; hence $q_{3} \in Q \cap A$ since $A$ is a $k$-ideal of $R$. Therefore, $\overline{q_{1}} \oplus \overline{q_{2}} \in A / I$. Now it is 
enough to show that if $r+I \in R / I$ and $a+I \in A / I$ (where $r \in Q, a \in A \cap Q$ ), then $(r+I) \odot(a+I) \in A / I$. There exists the unique element $q_{4} \in Q$ such that $(r+I) \odot(a+I)=q_{4}+I$ and $r a+I \subseteq q_{4}+I$, so $r a+c=q_{4}+d \in A$ for some $c, d \in I$. It follows that $q_{4} \in A \cap Q$; hence $q_{4}+I \in A / I$. Thus $A / I$ is an ideal of $R / I$.

Finally, assume that $t+I \in A / I$ and $(t+I) \oplus(s+I)=u+I \in A / I$ where $t, u \in A \cap Q, s \in Q$ and $t+s+I \subseteq u+I$. Then $t+s+c=u+d \in A$ for some $c, d \in I$; thus $s \in A \cap Q$ since $A$ is a $k$-ideal of $R$. Therefore, $s+I \in A / I$, as needed.

TheOREm 2.3. Let $R$ be a semiring, $I$ a $Q$-ideal of $R$ and $L$ a $k$-ideal of $R / I$. Then $L=J / I$ for some $k$-ideal $J$ of $R$.

Proof. Assume that $q^{\prime}$ is the unique element in $Q$ such that $q^{\prime}+I$ is the zero in $R / I$ and set $J=\left\{r \in R: q_{1}+I \in L\right\}$ (note that if $r \in R$, then there exists the unique element $q_{1} \in Q$ such that $\left.r \in q_{1}+I\right)$. We show that $J$ is a $k$-ideal of $R$ and $L=J / I$. The proof can now be broken down into a sequence of steps.

1) $I \subseteq J$. Let $a \in I$. By [3, Lemma 36], $a \in I=q^{\prime}+I \in L$, so $a \in J$. Thus $I \subseteq J$.

2) $J$ is an ideal of $R$. For if $r$ and $s$ are in $J$, then there are elements $q_{1}, q_{2} \in Q$ such that $q_{1}+I, q_{2}+I \in L, r=q_{1}+c$ and $s=q_{2}+d$ for some $c, d \in I$, so $\left(q_{1}+I\right) \oplus\left(q_{2}+I\right)=q_{3}+I \in L$ where $q_{3} \in Q$ is the unique element such that $q_{1}+q_{2}+I \subseteq q_{3}+I$; hence $r+s \in q_{1}+q_{2}+I \subseteq q_{3}+I \in L$. Thus $r+s \in J$. Similarly, if $r \in J$ and $t \in R$, then $r t=t r \in J$.

3) $J$ is a $k$-ideal of $R$. Let $a, a+b \in J$. Then there are elements $q_{1}, q_{2}$ and $q_{3}$ of $Q$ such that $a \in q_{1}+I \in L, a+b \in q_{2}+I \in L$ and $b \in q_{3}+I$, so $a=q_{1}+c, a+b=q_{2}+d$ and $b=q_{3}+e$ for some $c, d, e \in I$; hence $a+b \in\left(q_{1}+q_{3}+I\right) \cap\left(q_{2}+I\right)$. There is a unique element $q_{4}$ of $Q$ such that $\left(q_{1}+I\right) \oplus\left(q_{3}+I\right)=q_{4}+I$ where $q_{1}+q_{3}+I \subseteq q_{4}+I$, so $a+b \in\left(q_{2}+I\right) \cap\left(q_{4}+I\right)$; hence $q_{2}=q_{4}$. Therefore, $q_{3}+I \in L$ since $L$ is $k$-ideal; hence $b \in J$. Thus, $J$ is a $k$-ideal of $R$. Finally, it is easy to see that $L=J / I=\{q+I: q \in J \cap Q\}$.

Lemma 2.4. Let $R$ be a semiring with an identity $1, I$ a $Q$-ideal of $R$ and $A$ a $k$-ideal of $R$ with $I \subseteq A$. Then the following hold:

(i) There is a unique element $q_{0}$ of $Q$ such that $q_{0}+I$ is an identity of $R / I$.

(ii) If $q_{0}+I$ is an identity of $R / I$ and $q_{0}+I \in A / I$, then $R / I=A / I$.

(iii) If $q_{1}+I$ is an invertible element of $R / I$ with $q_{1}+I \in A / I$, then $R / I=A / I$.

Proof. (i) By assumption, there is a unique element $q_{0}$ of $Q$ such that $1=q_{0}+a$ for some $a \in I$. We show that $q_{0}+I$ is an identity of $R / I$. Let $q_{1}+I \in R / I$ for some $q_{1} \in Q$. Then there is a unique element $q_{2}$ of $Q$ such 
that $\left(q_{1}+I\right) \odot\left(q_{0}+I\right)=q_{2}+I$ and $q_{1} q_{0}+I \subseteq q_{2}+I$, so $q_{1} q_{0}+c=q_{2}+d$ for some $c, d \in I$. Since $q_{1}+c=q_{1} q_{0}+q_{1} a+c=q_{2}+q_{1} a+c+d \in\left(q_{1}+I\right) \cap\left(q_{2}+I\right)$, we must have $q_{1}=q_{2}$, as required.

(ii) Let $q_{1}+I \in R / I$ where $q_{1} \in Q$. Then $\left(q_{1}+I\right) \odot\left(q_{0}+I\right)=\left(q_{1}+I\right) \in A / I$ such that $q_{1} q_{0}+I \subseteq q_{1}+I$, so $q_{1} q_{0}+e=q_{1}+f \in A$ for some $e, f \in I$ since $q_{0} \in A$; hence $q_{1} \in A \cap Q$ since $A$ is $k$-ideal. Thus, $R / I=A / I$.

(iii) follows from (ii).

THEOREM 2.5. Let $R$ be a semiring, $I$ a $Q$-ideal of $R$ and $P$ a $k$-ideal of $R$ with $I \subseteq P$. Then $P$ is a prime ideal of $R$ if and only if $P / I$ is a prime ideal of $R / I$.

Proof. Let $P$ be a prime ideal of $R$. Suppose that $q_{1}+I, q_{2}+I \in R / I$ are such that $\left(q_{1}+I\right) \odot\left(q_{2}+I\right) \in P / I$ where $q_{1}, q_{2} \in Q$. Then there is a unique element $q_{3} \in Q \cap P$ such that $q_{1} q_{2}+I \subseteq q_{3}+I \in P / I$, so $q_{1} q_{2} \in P$. Then $P$ prime gives either $q_{1} \in P$ or $q_{2} \in P$; hence either $q_{1}+I \in P / I$ or $q_{2}+I \in P / I$ by Proposition 2.2 .

Conversely, suppose that $P / I$ is prime. Let $a, b \in R$ such that $a b \in P$. Then there are elements $q_{1}, q_{2} \in Q$ such that $a \in q_{1}+I$ and $b \in q_{2}+I$, so $a=q_{1}+c$ and $b=q_{2}+d$ for some $c, d \in I$. Since $a b=q_{1} q_{2}+q_{1} d+c q_{2}+c d \in P$ and $P$ is a $k$-ideal of $R$, we must have $q_{1} q_{2} \in P$. Let $q$ be the unique element in $Q$ such that $\left(q_{1}+I\right) \odot\left(q_{2}+I\right)=q+I$ where $q_{1} q_{2}+I \subseteq q+I$, so $q+e=q_{1} q_{2}+f$ for some $e, f \in I$. Since $P$ is a $k$-ideal of $R$, we get $q \in Q \cap P$ and $q+I \in P / I$; hence $P / I$ prime gives either $q_{1}+I \in P / I$ or $q_{2}+I \in P / I$. It follows that either $q_{1} \in P\left(\right.$ so $a \in P$ ) or $q_{2} \in P$ (so $b \in P$ ). Thus $P$ is prime.

THEOREM 2.6. Let $I$ be a proper $Q$-ideal of a semiring $R$. Then $I$ is prime if and only if $R / I$ is semidomain.

Proof. Let $q^{\prime}$ be the unique element in $Q$ such that $q^{\prime}+I$ is the zero in $R / I$. Let $I$ be a prime ideal of $R$ and let $q_{1}+I$ and $q_{2}+I$ be elements of $R / I$ such that $\left(q_{1}+I\right) \odot\left(q_{2}+I\right)=q^{\prime}+I$ where $q_{1}$ and $q_{2}$ are elements of $Q$. If $q_{1} \in I$, then $q_{1} \in\left(q^{\prime}+I\right) \cap\left(q_{1}+I\right)$ by [3, Lemma 36]; hence $q_{1}+I=q^{\prime}+I$. So suppose that $q_{1} \notin I$. By assumption, $q_{1} q_{2}+I \subseteq q^{\prime}+I$, so $q^{\prime}+e=q_{1} q_{2}+f \in q^{\prime}+I=I$ and [6, Lemma 2] gives $q_{1} q_{2} \in I$. Since $I$ is prime, we must have $q_{2} \in I=q^{\prime}+I$; hence $q_{2} \in\left(q_{2}+I\right) \cap\left(q^{\prime}+I\right)$, and it follows that $q_{2}+I=q^{\prime}+I$. Thus $R / I$ is semidomain.

Conversely, assume that $R / I$ is a semidomain and let $r, s \in R$ such that $r \notin I$ and $r s \in I$. By [3, Lemma 36], we must have $r \notin q^{\prime}+I=I$ and $r s \in q^{\prime}+I$. There are elements $q_{1}, q_{2} \in Q$ such that $r+I \subseteq q_{1}+I$ and $s+I \subseteq q_{2}+I$ since $I$ is a $Q$-ideal. Therefore, $r=q_{1}+a$ and $s=q_{2}+b$ for some $a, b \in I$. As $r \in q_{1}+I$ and $r \notin q^{\prime}+I$, we get $q_{1}+I \neq q^{\prime}+I$. It is easy to check that, $r s \in q_{1} q_{2}+I$. Let $q_{3}$ be the unique element in $Q$ such that $q_{1} q_{2}+I \subseteq q_{3}+I$. Since $r s \in\left(q^{\prime}+I\right) \cap\left(q_{3}+I\right)$, we must have $q^{\prime}=q_{3}$ 
and $\left(q_{1}+I\right) \odot\left(q_{2}+I\right)=q^{\prime}+I$; hence $s \in q_{2}+I=q^{\prime}+I=I$ since $R / I$ is semidomain. Thus $I$ is prime.

Corollary 2.7. Let $R$ be a semiring. Then $\{0\}$ is prime if and only if $R$ is semidomain.

Proof. This follows from Theorem 2.5 since $\{0\}$ is a $Q$-ideal of $R \cong R /\{0\}$ with $Q=R$.

Let $R$ be a semiring. We define a proper ideal $A$ of $R$ to be weakly primary (resp. weakly prime) if $0 \neq a b \in A$ implies $a \in A$ or $b^{m} \in A$ for some positive integer $m$ (resp. $a \in A$ or $b \in A$ ). So a primary ideal (resp. prime ideal) is a weakly primary (resp. weakly prime). However, since 0 is always weakly primary (resp. weakly prime) by definition, a weakly primary ideal (a weakly prime ideal) need not be primary (resp. prime). Clearly, every weakly prime is weakly primary (see [1] and [4]).

TheOREm 2.8. Let $R$ be a semiring, $I$ a $Q$-ideal of $R$ and $P$ a $k$-ideal of $R$ with $I \subseteq P$. Then the following hold:

(i) If $P$ is a weakly primary ideal of $R$, then $P / I$ is a weakly primary ideal of $R / I$.

(ii) If $I$ and $P / I$ are weakly primary, then $P$ is weakly primary.

Proof. (i) Let $q^{\prime}$ be the unique element in $Q$ such that $q^{\prime}+I$ is the zero in $R / I$. Assume that $P$ is weakly prime and let $q_{1}+I$ and $q_{2}+I$ be elements of $R / I$ such that $\left(q^{\prime}+I\right) \neq\left(q_{1}+I\right) \odot\left(q_{2}+I\right) \in P / I$, so there is a unique element $q_{3} \in Q \cap P$ with $q^{\prime} \neq q_{3}$ and $q_{1} q_{2}+I \subseteq q_{3}+I$; hence $0 \neq q_{1} q_{2} \in P$ since $P$ is a $k$-ideal. Then $P$ weakly primary gives either $q_{1} \in P$ or $q_{2}^{n} \in P$ for some $n$. If $q_{1} \in P$, then $q_{1}+I \in P / I$ by Proposition 2.2. So suppose that $q_{2}^{n} \in P$. Since $q_{2} \in q_{2}+I$, it is clear that $q_{2}^{n} \in\left(q_{2}+I\right)^{n}$. Thus $q_{2}^{n} \in\left(q_{2}+I\right)^{n} \cap q_{2}^{n}+I$, it follows that $\left(q_{2}+I\right)^{n}=q_{2}^{n}+I \in P / I$. Therefore, $P / I$ is weakly primary.

(ii) Let $0 \neq a b \in P$ where $a, b \in R$. If $a b \in I$, then $I$ weakly primary gives either $a \in I \subseteq P$ or $b^{s} \in I \subseteq P$ for some $s$. So we may suppose that $a b \notin I$. There are elements $q_{1}, q_{2} \in Q$ such that $a \in q_{1}+I$ and $b \in q_{2}+I$, so $a=q_{1}+c$ and $b=q_{2}+d$ for some $c, d \in I$. As $a b=q_{1} q_{2}+q_{1} d+c q_{2}+c d$, we must have $q_{1} q_{2} \in P \cap Q$ since $P$ is a $k$-ideal of $R$. Let $\left(q_{1}+I\right) \odot\left(q_{2}+I\right)=q_{4}+I$ where $q_{4} \in Q$ is the unique element such that $q_{1} q_{2}+I \subseteq q_{4}+I$. It follows that $q_{4} \in P \cap Q$; hence either $q_{1}+I \in P / I$ or $\left(q_{2}+I\right)^{m}=q_{2}^{m}+I \in P / I$ for some $m$ since $P / I$ is weakly primary. If $q_{1}+I \in P / I$, then $a=q_{1}+c \in P$ by Proposition 2.2. If $q_{2}^{m}+I \in P / I$, then $b^{m}=\left(q_{2}+d\right)^{m}=q_{2}^{m}+k \in P$ for some $k \in P$. Thus $P$ is weakly primary.

Corollary 2.9. Let $R$ be a semiring, $I$ a $Q$-ideal of $R$ and $P$ a $k$-ideal of $R$ with $I \subseteq P$. Then the following hold:

(i) If $P$ is a weakly prime ideal of $R$, then $P / I$ is a weakly prime ideal of $R / I$. 
(ii) If $I$ and $P / I$ are weakly prime, then $P$ is weakly prime.

Proof. This follows from Theorem 2.8.

A proper ideal $I$ in a semiring $R$ is said to be maximal (resp. $k$-maximal) if $J$ is an ideal (a $k$-ideal) in $R$ such that $I \varsubsetneqq J$, then $J=R$.

THEOREM 2.10. Let $I$ be a proper $Q$-ideal of a semiring $R$ with an identity 1. If $I$ is a maximal ideal of $R$, then $R / I$ is a semifield.

Proof. Assume that $q^{\prime} \in Q$ is the unique element in $Q$ such that $q^{\prime}+I$ is the zero in $R / I$ and let $q_{0}+I$ be the identity element of $R / I$ by Lemma 2.4 where $1=q_{0}+a$ for some $a \in I$ and the unique element $q_{0} \in Q$. It suffices to show that every non-zero element $q_{1}+I$ of $R / I$ is invertible. As $I$ is a proper ideal of $R$, we must have $q_{0} \notin I$, so $q_{1} \notin I$; hence $I+R q_{1}=R$ by maximality of $I$. There exist $r \in R$ and $a \in I$ with $r q_{1}+a=1$. As $I$ is a $Q$-ideal of $R$, $r=q_{2}+c$ for some $q_{2} \in Q$ and $c \in I$, so $q_{1} q_{2}+c q_{1}+a=1 \in q_{1} q_{2}+I$. Since $\left(q_{1}+I\right) \odot\left(q_{2}+I\right)=q_{3}+I$ where $q_{3} \in Q$ is the unique element such that $q_{1} q_{2}+I \subseteq q_{3}+I$, we must have $1 \in\left(q_{0}+I\right) \cap\left(q_{3}+I\right)$; hence $q_{3}=q_{0}$, and the proof is complete.

THEOREM 2.11. Let $I$ be a proper $Q$-ideal of a semiring $R$ with an identity 1. If $R / I$ is a semifield, then $I$ is a $k$-maximal ideal of $R$.

Proof. Suppose that $q^{\prime} \in Q$ is the unique element in $Q$ such that $q^{\prime}+I$ is the zero in $R / I$ and let $q_{0}+I$ be the identity element of $R / I$ by Lemma 2.4 where $1=q_{0}+a$ for some $a \in I$ and the unique element $q_{0}$ of $Q$. Assume that $R / I$ is a semifield and $I \varsubsetneqq J$ for some $k$-ideal $J$ of $R$; we show that $J=R$. Then there are elements $t \in Q$ and $c \in J-I$ with $c \in t+I$, so $c=t+d$ for some $d \in I$; hence $t \in J-I$. If $t+I=q^{\prime}+I=I$, then $t=q^{\prime} \in I$ which is a contradiction. Therefore, there exists $s+I \in R / I$ such that $(t+I) \odot(s+I)=q_{0}+I$; thus $s t+e=q_{0}+f$ for some $e, f \in I$. It follows that $s t+a+e=q_{0}+a+f=1+f \in J$, so $1 \in J$ since $J$ is a $k$-ideal. Thus $J=R$, as required.

Lemma 2.12. Let $R$ be a semiring. Then the following hold:

(i) If $I$ and $J$ are $k$-ideals of $R, I+J$ is a $k$-ideal of $R$.

(ii) An intersection of a family of $k$-ideals of $R$ is $k$-ideal.

PROOF. The proof is completely straightforward.

Lemma 2.13. Assume that $I$ is a $Q$-ideal of a semiring $R$ and let $J, L$ be $k$-ideals of $R$. Then the following hold:

(i) $(I+J) / I$ is a $k$-ideal of $R / I$.

(ii) If $I \subseteq J$ and $I \subseteq L$, then $J / I=L / I$ if and only if $J=L$. 
Proof. (i) Since by [6, Lemma 2], every $Q$-ideal is $k$-ideal, we must have $I+J$ is $k$-ideal by Lemma 2.12 ; hence $(I+J) / I$ is a $k$-ideal of $R / I$ by Proposition 2.2.

(ii) Let $a \in J$. Then $a \in q_{1}+I$ for some $q_{1} \in Q$, so there is an element $c \in I \subseteq J$ such that $a=q_{1}+c$; hence $J k$-ideals gives $q_{1} \in J$. Thus $q_{1}+I \in J / I=L / I$, so $q_{1}+I=q_{2}+I$ for some $q_{2} \in L \cap Q$. It follows that $q_{1} \in L$ since $L$ is $k$-ideal. Therefore, $a \in L$; thus $J \subseteq L$. Similarly, $L \subseteq J$, as required.

Theorem 2.14. Let $R$ be a semiring, $I$ a $Q$-ideal of $R$ and $P$ a $k$-ideal of $R$ with $I \subseteq P$. Then $P$ is a k-maximal ideal of $R$ if and only if $P / I$ is a $k$-maximal ideal of $R / I$.

Proof. Assume that $P$ is a $k$-maximal ideal of $R$ and let $L$ be a $k$-ideal of $R / I$ such that $P / I \varsubsetneqq L$. It then follows from Theorem 2.3 that there exists a $k$-ideal $J$ of $R$ with $P / I \varsubsetneqq L=J / I$, so $P \varsubsetneqq J$ by Lemma 2.13 ; hence $J=R$. Thus $L=R / I$. The other implication is similar.

If $R$ is a semiring, then $R$ is Noetherian (resp. Artinian) if any non-empty set of $k$-ideals of $R$ has a maximal (resp. minimal member) with respect to set inclusion. This definition is equivalent to the ascending chain condition (resp. descending chain condition) on $k$-ideals of $R$.

TheOREM 2.15. Let I be a $Q$-ideal of a semiring $R$. Then the following hold:

(i) If the semiring $R$ is Noetherian, then $R / I$ is a Noetherian semiring.

(ii) If the semiring $R$ is Artinian, then $R / I$ is a Artinian semiring.

Proof. (i) By Theorem 2.3, an ascending chain of $k$-ideals of $R / I$ must have the form

$$
J_{1} / I \subseteq J_{2} / I \subseteq \cdots \subseteq J_{n} / I \subseteq J_{n+1} / I \subseteq \cdots
$$

where

$$
J_{1} \subseteq J_{2} \subseteq \cdots \subseteq J_{n} \subseteq J_{n+1} \subseteq \cdots
$$

is an ascending chain of $k$-ideals of $R$ all of which contain $I$. Since the latter chain must eventually become stationary, so must the former by Lemma 2.13.

(ii) This can be proved in a very similar manner to the way in which (i) was proved above, and we omit it.

TheOREM 2.16. Let I be a $Q$-ideal of a semiring $R$. Then the following hold:

(i) If both $I$ and $R / I$ are Noetherian, then $R$ is Noetherian.

(ii) If both $I$ and $R / I$ are Artinian, then $R$ is Artinian. 
ProOF. (i) Let

$$
J_{1} \subseteq J_{2} \subseteq \ldots \subseteq J_{n} \subseteq J_{n+1} \subseteq \cdots
$$

be an ascending chain of $k$-ideals of $R$. Then Lemma 2.12 gives

$$
J_{1} \cap I \subseteq J_{2} \cap I \subseteq \cdots \subseteq J_{n} \cap I \subseteq J_{n+1} \cap I \subseteq \cdots
$$

is an ascending chain of $k$-ideals of $I$, and so there is a positive integer $s$ such that $J_{s} \cap I=J_{s+i} \cap I$ for all positive integer $i$. By Lemma 2.13,

$$
\left(J_{1}+I\right) / I \subseteq\left(J_{2}+I\right) / I \subseteq \cdots \subseteq\left(J_{n}+I\right) / I \subseteq\left(J_{n+1}+I\right) / I \subseteq \cdots
$$

is a chain of $k$-ideals of $R / I$. Thus there exists a positive integer $t$ such that $\left(J_{t}+I\right) / I=\left(J_{t+i}+I\right) / I$ for all positive integer $i$, so that $I+J_{t}=I+J_{t+i}$ for all $i$. Let $u=\max \{s, t\}$. We show that, for each positive integer $i, J_{u}=J_{u+i}$. Since the inclusion $J_{u} \subseteq J_{u+i}$ is trivial, we will prove the reverse inclusion. Let $x \in J_{u+i}$. Since $x \in I+J_{u+i}=I+J_{u}$, we must have $x=a+b$ for some $a \in I$ and $b \in J_{u} \subseteq J_{u+i}$. Hence $a \in J_{u+i}$ since it is $k$-ideal. It follows that $a \in I \cap J_{u+i}=I \cap J_{u}$; hence both $a$ and $b$ belong to $J_{u}$ and $x \in J_{u}$, as needed.

(ii) This proof is similar to that in the case (i) and we omit it.

ACKNOWLEDGEMENTS.

The author thanks the referee for useful comments.

\section{REFERENCES}

[1] D. D. Anderson and E. Smith, Weakly prime ideals, Houston J. of Math. 29 (2003), 831-840.

[2] P. J. Allen, A fundamental theorem of homomorphisms for semirings, Proc. Amer. Math. Soc. 21 (1969), 412-416.

[3] P. J. Allen and J. Neggers, Ideal theory in commutative semirings, Kyungpook Math. J. 46 (2006), 261-271.

[4] S. Ebrahimi Atani and F. Farzalipour, On weakly primary ideals, Georgian Math. J. 12 (2005), 423-429.

[5] V. Gupta and J. N. Chaudhari, Some remarks on semirings, Radovi Matematicki 12 (2003), 13-18.

[6] V. Gupta and J. N. Chaudhari, Right $\pi$-regular semirings, Sarajevo J. of Math. 14(2) (2006), 3-9.

[7] J. R. Mosher, Generalized quotients of hemirings, Compositio Math. 22 (1970), 275281.

S. E. Atani

Department of Mathematics

University of Guilan

P.O. Box 1914

Rasht

Iran

Received: 11.9.2006.

Revised: 28.11.2006. 